\title{
Wireless Sensor Network Simulators Relevance compared to a real IEEE 802.15.4 Testbed
}

\author{
Stéphane Lohier, Abderrezak Rachedi, Erwan Livolant, Ismail Salhi \\ Computer Science Laboratory (LIGM) \\ University of Paris-Est Marne-la-Vallee (UPEMLV), France \\ \{lohier, rachedi, livolant, salhi\}@univ-mlv.fr
}

\begin{abstract}
The diversity of research topics in Wireless Sensor Networks (WSNs) is attracting more and more researchers from different fields. The common point of all proposed solutions and protocols for WSNs is the evaluation usually done with network simulators. In this paper, we focus on the results relevance of wireless sensor network simulators in different scenarios based on indoor and outdoor environments. We propose a comparative study between 3 usual simulators (NS2, OPNET and QualNet) while using as reference a real testbed based on recent Imote 2 sensors. The simulators give different results even in similar environments. NS2 and Qualnet give results close to those of the experimentation in the case of an indoor environment, but in the outdoor environment Opnet gives results closer to the reality. In addition, the impact of different MAC protocols (B-MAC and TKN15.4 $M A C)$ which observe or do not observe the IEEE 802.15.4 standard is illustrated by real experimentations. They show that TKN15.4 protocol gives a better throughput than B-MAC.
\end{abstract}

Keywords: Wireless Sensor Networks, IEEE 802.15.4, Network Simulators, Testbed

\section{INTRODUCTION}

The design and the implementation of new protocols or models for wireless sensor networks are mainly based on simulation in order to test and validate the proposed solutions. Simulations are indeed less costly and complex than a real implementation in a testbed, especially if the density of the network and the complexity of the topology used are significant. Moreover, we are able to compare the performances of different protocols/models in different scenarios and through different periods much faster with simulations. When implementing a real WPAN testbed with ZigBee [1] devices, we have to take into account the environment type, the terminal location and the MAC sub-layer parameters as they may have an important impact on the different protocols/models performances. To assess the relevance of a specific wireless network simulator, two questions have to be answered: 1) Is the implementation of the lower layers, specially the physical layer, close to the reality? 2) If the implementation is not enough realistic, how can we evaluate the relevance of our results, particularly in the case of a dense multi-hop network, as we know that the transmission model between two nodes does not match a real situation?

Our involvement in such a study is thus due to three observations we made: 1) No work dealing with the latest 802.15.4 [2] devices ever reported any evaluation of their performance and observance of the IEEE standard. These studies focused on the different MAC 802.15.4 implementations (B-MAC, TinyOS 15.4 WG, TinyOS ZigBee
WG, etc.) but we are not aware of any comparative study between the devices. 2) No work proposed a recent IEEE 802.15.4 comparative study between simulators. 3) No work studies the simulator relevance in comparison with real 802.15.4 testbed.

This paper thus aims at:

1) Assessing the performances of different MAC layers implemented in the latest 802.15.4 devices based on the CC2420 RF transceiver [11] and checking their observance of the IEEE standard. The 802.15.4 devices performances are evaluated both in an indoor and in an outdoor environment. 2) Estimating the relevance of the MAC 802.15.4 layer lately implemented in the different network simulators and comparing it to a real testbed. We will thus be able to provide researchers a guideline to help them choose and tune a 802.15.4 simulator in accordance with a specific environment.

To achieve these goals, we use four different environments: an outdoor free-space and three indoor environments (office, corridor and hall). For the indoor-office environment, we first study a simple transmission without contentions between two nodes, and then transmissions with one to seven emitters in competition to access to the media. The comparisons are made with three major network simulators: NS2 [14], OPNET [15] and QualNet [16]. For the testbed, two MAC protocols are investigated: B-MAC (Berkeley MAC) [19] which does not observe the IEEE 802.15.4 standard and TKN15.4 MAC [18] which does. B-MAC protocol is a native protocol of many hardware devices like Crossbow sensors (MICAz, TelosB, Imote2). TKN15.4 MAC protocol includes almost the complete functionality described in the 802.15.4-2006 [2] specification, except for GTS allocation and management, security services, and a few minor services like PAN ID conflict notification.

This paper is divided into five sections. In section 2 , we sum up the existing works dealing with experimentation results and network simulators. We present an evaluation of B-MAC and TKN15.4 MAC over Imote2 in section 3. Section 4 is dedicated to the comparative study of the major network simulators performances. The fifth section concludes our paper and presents the future works.

\section{RELATED WORKS}

Many works in literature deal with the performance evaluation of the IEEE 802.15.4 standard. These studies can be divided into three categories. In the first one [3-4], the results are obtained with real 802.15.4 devices. In the second one [57], they are obtained with different simulations. In the third one 
[8-10], the analysis and modelling of the 802.15.4 channel enable the network performances assessment.

The complete study proposed by Petrova et al. [3] analyzes the performance of IEEE 802.15.4 through the measurement of the PER (Packet Error Rate) and the RSSI (Received Signal Strength Indicator) both in indoor and outdoor environments with only one emitter and one receiver using the usual CC2420 RF transceiver. The results demonstrate the OQPSK modulation efficiency compared to other schemes included in $\mathrm{WiFi}$ or Bluetooth technologies. The results are then used to tune the error model in NS-2 simulator [14] for the IEEE 802.15.4 MAC extension [12]. The simulations show that in the slotted CSMA-CA mode, the throughput is always lower than $45 \mathrm{kbps}$ (for a $250 \mathrm{kbps}$ nominal raw bit rate), whatever the offered load and the number of sources (from 1 to 60). The conclusion is that the beaconed mode should only be used when the resulting short channel access time is critical, and the offered load is low. No comparison on the throughput is done between the simulator and the testbed.

In a similar study [4], the authors study the performance of the direct and indirect data transmissions using an IEEE 802.15.4 compliant CC2420 transceiver in the CSMA/CA beacon-enabled mode and analyze the effects of the data payload size. This study shows that: (1) the data throughput is significantly reduced in the case of indirect transmissions (from $153 \mathrm{kbps}$ to $65 \mathrm{kbps}$ for a $250 \mathrm{kbps}$ raw bit rate); (2) when the number of sources increases, both the effective data rate and delivery ratio decrease because of collisions and random backoffs; (3) the data rate increases according to the payload size but it has no significant influence on the delivery ratio.

In [5], Lu et al. evaluate the performances of 802.15.4 MAC for the beacon-enabled mode, using NS-2 simulations. They found that an extremely low duty cycle operation enables significant energy savings but can lead to an important latency and a low bandwidth. The CSMA-CA algorithm reduces the energy costs but increases the number of collisions at a higher rate and with a larger number of sources. However, the use of GTS (Guaranteed Time Slots) can ensure a low latency but increases the energy costs. Thus, it is difficult to find a tradeoff, which mainly depends on the context and the type of application (real time, high throughput...). In this study, no guideline is proposed to tune the parameters of the simulator.

Zheng et al. present in [6] a complete simulation set using the Samsung/CUNY 802.15.4 implementation in an NS-2 simulator [12]. This study shows that the 802.15.4 standard suffers from hidden terminal problems because of the absence of any RTS/CTS mechanism. However, for low data rates (up to one packet per second), the performance decrease is minor. The default CSMA-CA backoff period is too short, which leads to frequent repeated collisions. The superframes with low beacon orders (short duration between beacons) can also lower the slotted CSMA-CA backoff efficiency and lead to a high collision probability when the superframes are launched.

In [7], the authors have developed simulation tools for IEEE 802.15.4 slotted CSMA/CA mechanism using an OPNET simulator [15]. The sensor network is composed of a PAN coordinator and 100 nodes randomly spread in a $100 \mathrm{~m} \mathrm{x}$ $100 \mathrm{~m}$ area. The basic conclusions are that the backoff algorithm is not flexible enough for large-scale sensor networks; the offered load (corresponding to the inter-arrival times of the flows in each node) should be around 50\% to offer the best trade-off between throughput and average delay; lower superframe orders (active duration of the superframe) introduce additional overheads and thus limit the throughput.

The performance of the IEEE 802.15.4 contention access period is also analyzed in [8] in terms of throughput and energy consumption. For the analysis, the behavior of the nodes and the channel are modeled, using Markov chains. For the lower layers, the authors used the characteristics of the CC2420 transceiver. They show that the standard specified MAC can be accurately modeled as non-persistent CSMA. They also demonstrate that letting the radio in a shutdown state between the different transmissions is a very effective means of reducing the average power consumption for a very wide range of traffic rates. Finally, they propose to initialize the contention window length to 1 , in order to significantly improve the throughput and reduce the energy consumption when MAClevel acknowledgements are not used.

Misic et al. have modeled in [9] the operation of the IEEE 802.15.4 MAC layer in the beacon-enabled mode through discrete time Markov chains. They identify the downlink queue stability at the PAN coordinator as the tightest criterion for the network. Consequently, they assume that the number of nodes and their traffic load should be chosen to avoid the saturation point of the network.

In [10], the authors provide an analytic performance model using Markov chains in order to compute the saturation throughput of the network in a star topology. The model is validated through simulations with NS2 in a network composed of a maximum of 50 nodes. One of the main conclusions is that the aggregated throughput is never higher than $70 \mathrm{Kbps}$, whatever the number of nodes and the total load.

As far as we know, no comparison between WPAN 802.15.4 simulators and real testbed has ever been carried out. The added value of our study is thus to present and analyse a complete set of measurements (throughput, RSSI and loss ratio) with a recent 802.15.4 testbed and then to compare the testbed and the simulators' results in order to point out their divergences and the causes of these divergences.

\section{EVALUATION OF MAC LAYERS OVER IMOTE2}

\section{A. Context}

In this section, we point out the significant impact of the environment on wireless communications. The indoor environment is more common (companies, offices, home, etc). The outdoor environment represents a free-space area without any physical obstacle, such as emergency deployments. It is thus much less frequent, especially in a WPAN context. Nevertheless, many performance simulation studies on WPAN use the free-space or the two-ray ground propagation models, whatever the supposed context. These models are obviously not adapted to an indoor environment. The free-space model does not take into account the floor reflection signals and the two-ray ground model takes these signals into account from a certain distance threshold between the transmitter and the receiver. 
For our experiment, we selected four realistic environments. The first one is an outdoor environment corresponding to our campus park without any obstacle (freespace). The three others are indoor and take place in our laboratory building. As the WiFi technology is currently used in all buildings, we kept the existing IEEE $802.11 \mathrm{~g}$ communication on, in order to get results as close to the reality as possible. When the 802.15.4 sensor wants to send a packet, it selects an available channel. The first indoor environment is the most usual one: it is represented by our laboratory, made up of $15-30 \mathrm{~m} 2$ offices located along a corridor (figure 1). For this indoor-office environment, we use a simple scenario with a fixed emitter and a mobile receiver in order to evaluate the range and the fading effects across several offices (each office is 3 meter long and is separated from the next one by a concrete wall $30 \mathrm{~cm}$ thick). The objective here is to characterize and compare the different MAC layers existing for the CC2420 transceiver, another scenario with several emitters creating contention is presented in next section.

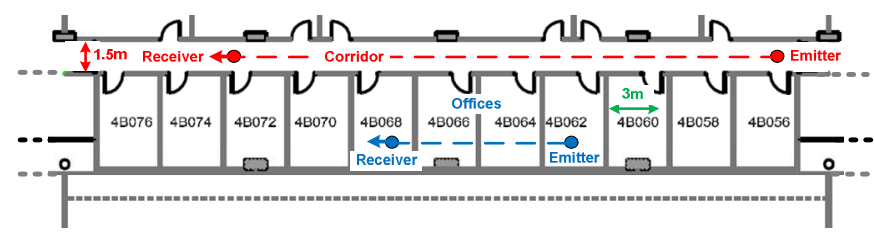

Figure 1. The indoor environment (Paris-Est computing research laboratory)

The second indoor environment is the corridor also shown in figure 1. The goal is to highlight the corridor effect which theoretically allows higher ranges. The third indoor environment is the hall of our building, an open space with a ten meter high ceiling and a length of eighty meter in its longest part. This place is comparable with station or airport halls.

In both scenarios, we used one CBR connection with the maximum rate in order to reach the limit of the channel capacity $(1 \mathrm{kHz})$. The selected frame size is set to its maximum value (127 Bytes). The transmitter and receiver nodes are placed at a height of $1 \mathrm{~m}$ corresponding to a desk height. The distance between them fluctuates between 0 and $65 \mathrm{~m}$, which is almost the maximum range except for the corridor.

TABLE I. CC2420 CHARACTERISTICS

\begin{tabular}{|l|l|}
\hline Frequency Band (ISM) & $2400.0-2483.5 \mathrm{MHz}$ \\
\hline Data Rate & $250 \mathrm{~kb} / \mathrm{s}$ \\
\hline Tx Power & $-24-0 \mathrm{dBm}$ \\
\hline Rx Sensitivity & $-94 \mathrm{dBm}$ \\
\hline Range (line of sight) & $\sim 30 \mathrm{~m}$ \\
\hline
\end{tabular}

The nodes used in the testbed are the recent MEMSIC (formerly Crossbow) [13] Imote2 equipped with CC2420 radio transceiver (see table I). The native MAC protocol in Imote2 sensors (BMAC) does not observe IEEE 802.15.4 standard and many capabilities, such as the beacon-enabled mode, are not implemented. In order to compare BMAC and other protocols in conformity with the standard, we have adapted the TKN15.4 MAC protocol implementation provided by the TinyOS 15.4 working group [17] to the Imote 2 platform. In this study, we only focus on the non-beacon-enabled mode, in order to provide a fair comparison between TKN-15.4 and BMAC. The selected metrics are the throughput, the RSSI (Received Signal Strength Indication) and the packet loss ratio in order to evaluate the performance of the transmission and the quality of the channel.

\section{B. Results}

The results related to the average throughput assessed at the receiver according to the different distances between the sender and the receiver are plotted in figures 2 and 3. Both scenarios (outdoor-free-space, indoor-office, indoor-corridor and indoorhall) and the three MAC layers (BMAC, TKN-15.4, and TKN15.4 with $\mathrm{ACK}$ ) are represented. Whatever the context, the TKN-15.4 MAC layer gives a better result than BMAC in terms of throughput. The TKN-15.4 enhances the throughput up to $50 \%$ compared to BMAC. This result reflects the different implementations of both MAC protocols in terms of buffer management policy and backoff algorithms. Except in the case of BMAC, the throughput reaches $50 \%$ of the raw bit rate $(250 \mathrm{kbps})$. In addition, we can compare the throughput provided by the same MAC layer (TKN-15.4) in both acknowledgement-enabled and non-acknowledgement enabled modes. This comparison shows that the acknowledgementenabled mode is more reliable thanks to the retransmission mechanism, whereas the non-acknowledgement-enabled mode provides a $10 \%$ higher capacity (see also figure 6 and 7).

In the outdoor scenario (line-of-sight), the measured range is about the triple $(55 \mathrm{~m})$ of that obtained in an office environment $(15 \mathrm{~m})$. Until this value, the throughput in the outdoor scenario is almost constant. This result demonstrates the robustness of the 802.15.4 channel. In the indoor-office scenario, the throughput becomes null beyond $15 \mathrm{~m}$ (no more propagation) which correspond to 5 offices. Before this threshold, up to the 4th office, the throughput remains almost constant with weak variations proportional to the RSSI (see figure 4). Let us note that it is mainly the number of walls which limit the range, this one would be higher for longer offices. The range for the indoor-hall scenario $(65 \mathrm{~m})$ is a little higher than for the outdoor context $(55 \mathrm{~m})$ thanks to the reflections on the floor and the walls. These effects are much more important in the case of the corridor: the geometry of the places (1.6m between both walls) associated to a line-of-sight propagation favors a wave canalization effect. These reflections produce an important gain and increase the range up to $180 \mathrm{~m}$. Let us also note that the corridor effect produces, for a distance of $25 \mathrm{~m}$, a weak reduction of the throughput due to the superposition, at this point, of the direct signal (with a $2.4 \mathrm{GHz}$ carrier) and the signal reflected on the floor.

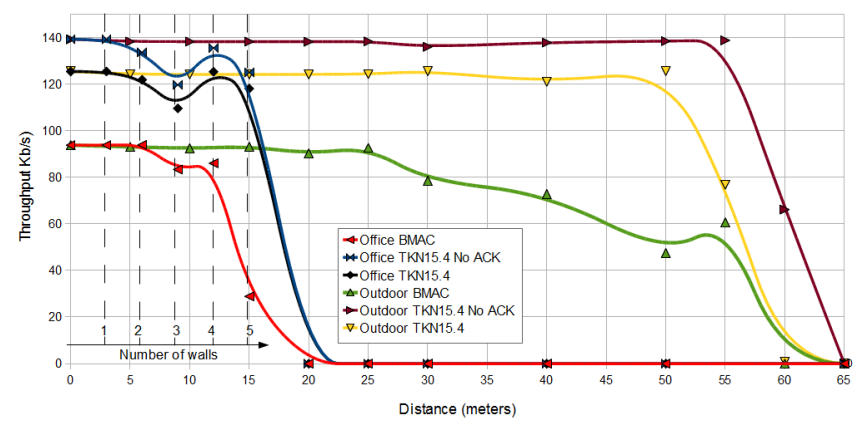

Figure 2. Throughput vs Distance (Office and Outdoor) 


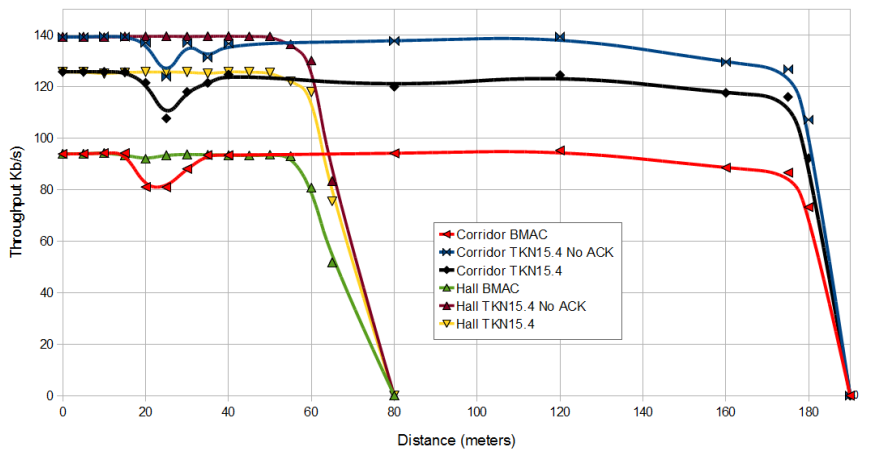

Figure 3. Throughput vs Distance (Corridor and Hall)

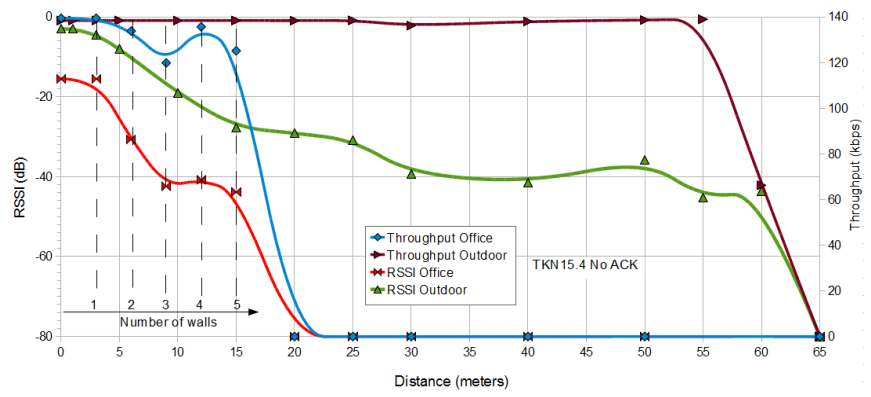

Figure 4. RSSI and Throughput vs Distance (Office and Outdoor)

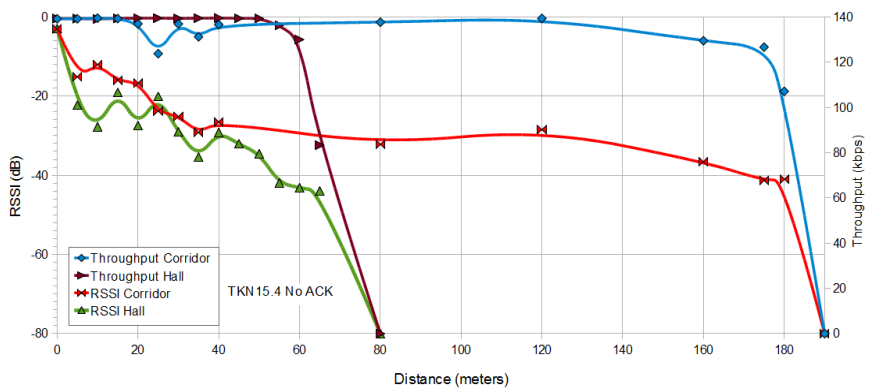

Figure 5. RSSI and Throughput vs Distance (Corridor and Hall)

Figures 4 and 5 show the evolution of the RSSI and throughput measured at the receiver according to the distance (only the TKN-15.4 MAC layer without ACK is plotted). In the cases of the outdoor and the indoor-hall scenarios, according to the mathematic formulation of free-space environment, the signal level should decrease according to the distance square. The measured signal level is more versatile than expected, particularly in the hall. It depends on many parameters, such as the characteristics and orientation of the antennas, the ground reflections, the potential background noise, etc. For the corridor, the RSSI remains almost constant between $40 \mathrm{~m}$ and $160 \mathrm{~m}$ which confirms the corridor effect due to the importance of the reflections. In the case of the office environment, we expected a significant fluctuation of the signal level because of the number and nature of the dividing walls, the people moving in the area, the interferences with other transmissions, etc. The results show that the signal level decreases starting from the 2nd wall and remains relatively constant up to the 5th wall, which also demonstrates the robustness of the channel. What is the impact of the RSSI fluctuations on the throughput? Figure 4 and 5 show that the throughput remains constant up to a certain threshold (from 15 to $160 \mathrm{~m}$, depending on the environment), even if the signal level decreases or fluctuates. The 802.15.4 device thus tries to maintain a high data rate until a certain received power threshold (approximately $-40 \mathrm{~dB}$ ).

The fluctuation of the packet loss ratio according to the distance is plotted in figures 6 and 7. The ratio is much higher for the BMAC in both contexts, which explains the differences in the measured throughputs. With the TKN-15.4 protocol, the loss rate remains very weak for ranges that are close to the limit. Even in the indoor-office environment, the rate remains lower than $15 \%$ until a $15 \mathrm{~m}$ distance (4 offices). For the corridor scenario, the corridor effect at $25 \mathrm{~m}$ is also illustrated in figure 7. Finally, the measured ratios enable us to give some guidelines for an IEEE 802.15.4 network deployment in both indoor and outdoor environments: the router mesh should have a 15-20m diameter in a noisy indoor environment and a 50-60 $\mathrm{m}$ diameter in a free-space environment.

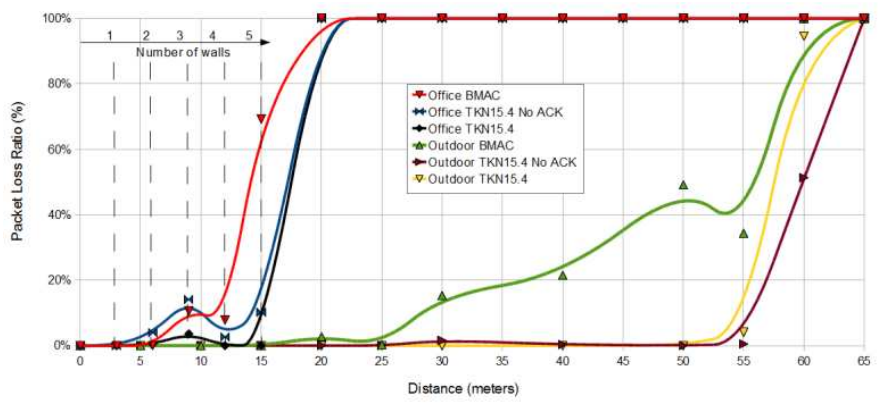

Figure 6. Packet Loss Ratio vs Distance (Office and Outdoor)

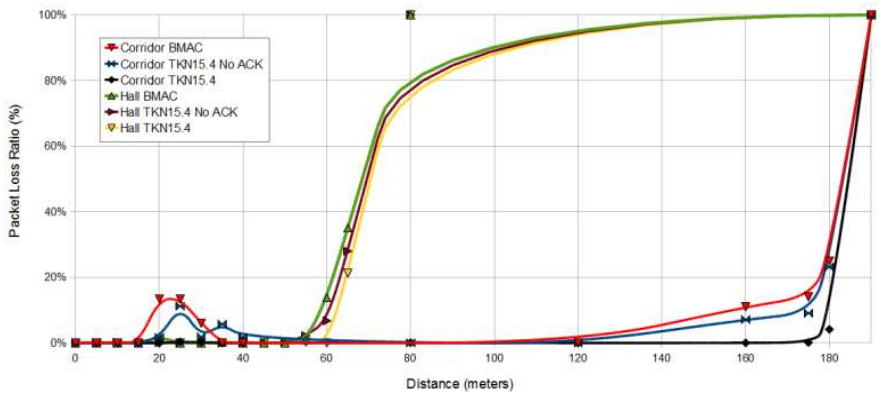

Figure 7. Packet Loss Ratio vs Distance (Corridor and Hall)

\section{COMPARISONS BETWEEN TESTBED AND SIMULATORS}

A. NS2

NS-2 [14] is currently the most popular network simulator. We used the Free-Space propagation models to simulate the outdoor environment and the Shadowing model with different parameters to simulate the indoor environment. In the latest versions of NS-2, the IEEE 802.15.4 extension developed at the City College of New York is included. Moreover, NS-2 observes the 802.15.4 standard and the frames are always acknowledged. The simulation parameters (frame size, CBR rate, data rate) are the same as those used for the testbed. The transmitted power is tuned in the free-space model in order to obtain a $55 \mathrm{~m}$ reception range. To be as close to the real testbed as possible, neither the background noise nor the interferences are taken into account in the vicinity of the nodes.

For the Free-Space model, the signal power attenuation is proportional to $1 / d^{2}$ : 


$$
P_{r}=\frac{P_{t} G_{t} G_{r} \lambda^{2}}{(4 \pi d)^{2} L}
$$

$G_{t}$ and $G_{r}$ are the antennas gains, $\lambda$ the wavelength, $L$ the Loss Factor and $d$ the distance between two nodes. We tuned the values of Transmitted Power $\left(P_{t}\right)$ and Capture Threshold $\left(P_{r}\right)$ to obtain the same range as in the real testbed. These reference values are also used for the other propagation models.

For the shadowing model, two important parameters are used to differentiate the environment:

$$
\left[\frac{P_{r}(d)}{P_{r}\left(d_{0}\right)}\right]_{d B}=-10 \beta \log \left(\frac{d}{d_{0}}\right)+X_{d B}
$$

where $d_{0}$ is a reference distance, $\beta$ is the path loss exponent and is usually empirically determined by a field measurement. For instance, $\beta=2$ corresponds to a free-space propagation. When we set $\beta$ to a larger value, that means that the number of obstructions is more significant, and the greater the distance, the faster the decrease of the received signal power. The second parameter $X_{d B}$ is a log-normal random variable which reflects the fluctuation of the received power at a certain distance. $X_{d B}$ is thus a Gaussian random variable with zero mean and a standard deviation $\sigma_{d B}$ which is called the shadowing deviation. $\sigma_{d B}$ is also obtained by measurement. For example, $\sigma_{d B}=7 \mathrm{~dB}$ corresponds to an obstructed office environment.

\section{B. Qualnet}

In order to carry out an efficient and fair comparison between the simulators, we used the same parameters as in NS2. The proposed propagation models are close to those proposed in NS-2: free-space and log-normal shadowing (in this model, only the deviation parameter is used). Qualnet also proposes the Rayleigh fading model, which occurs when there is no line of sight between the source and destination.

\section{OPNET}

The wireless suite of OPNET Modeler [16] integrates 802.15.4 devices (PHY Extended Rate) and offers 5 propagation models intended for outdoor environments (FreeSpace, Longley-Rice, Hata, CCIR, Walfisch-Ikegami). OPNET Modeler views all wireless channels as Gaussian channels (uniform noise spectral density) and ignores the fading effect. In addition, OPNET uses a fixed value of the pathloss exponent without considering the diversity of the environments. As for NS-2 and Qualnet the transmitted power is tuned in the freespace model in order to obtain a $55 \mathrm{~m}$ reception range. In that case, the obtained throughput is close to the reality.

\section{Results}

For these comparisons, we use three scenarios. Two scenarios (outdoor-free-space and indoor-office) with one fixed emitter and a mobile receiver, like previously. The third is located in an office with one receiver and from 1 to 7 transmitters in order to evaluate the impact of the contention on the global throughput. The transmitters are placed at $1 \mathrm{~m}$ from the receiver. Each transmitter is located at $10 \mathrm{~cm}$ from its neighbor.
The testbed results are used as reference to compare the performances of the three simulators, see figures 8,9 and 10 . First, we notice that the throughput obtained with NS-2 simulations is always higher, whatever the model. This is mainly due to the implementation of the 802.15.4 MAC layer. As for all simulators, the free-space model gives a binary response: beyond a certain threshold, no more packets will be received. The shadowing model offers results closer to the reality but the parameters that drive the shadowing propagation are difficult to set. The documentation of NS-2 gives typical values of $\beta$ and $\sigma$ (for example: $\beta=2$ and $\sigma=4$ to $12 \mathrm{~dB}$ for an outdoor free-space environment; $\beta=4$ to 6 and $\sigma=6.8 \mathrm{~dB}$ for an indoor obstructed environment) but the shadowing model is probabilistic and insofar as these parameters have to be determined by field measurement, it is difficult to reflect the reality. Thus, it is possible to tune the parameters in order to obtain a curve close to the reality (indoor or outdoor) but these configurations cannot be generalized. In figure 8 , for $\beta=2$ and $\sigma=4$, the throughput shows almost the same decrease as for the testbed in the indoor environment. However, according to the model, these parameters correspond to an "in building, line-ofsight" environment.

Concerning Qualnet, the first remark is related to the average values of the throughputs which are lower than those obtained with the testbed or the other simulators (in the indoor and outdoor environments). Once again, the implementation of the MAC layer (backoff algorithm, clear channel assessment, MAC buffers...) has a significant impact on the performance. As for NS-2, the shadowing model gives better results for the indoor environment, despite a lower throughput. The shadowing deviation is tuned in order to obtain a curve close to the reality $(\sigma=4 \mathrm{~dB})$ and the configuration is much more precise and effective than for NS.

The free-space model of OPNET gives results close to the reality. With the same power value, the other propagation models give very nearby results and are thus not represented. They are indeed designed for an outdoor environment and thus for transmission powers much higher than those usually used in IEEE 802.15.4 (greater than $100 \mathrm{~mW}$ ) and for antennas with a range much higher than this present in the nodes (greater than $100 \mathrm{~m}$ ). Although OPNET integrates 802.15.4 device and a lot of potential configurations (noise, loss factor, antenna models...), the propagation models proposed by default do not enable to carry out simulations close to the reality in an indoor environment (the HATA model plotted in figure 9 gives a binary response like for the free-space model). It is necessary in this case to add other propagation models, such as the shadowing model. In addition, the beacon-enabled mode is not implemented.

For the scenario with contention (figure 10), the shadowing model proposed in NS2, with optimized parameters $(\beta=2$ and $\sigma=4)$ gives results very close to those obtained with the testbed: the average global throughput increases slightly with the number of sources until a threshold close to $135 \mathrm{Kbps}$. For the other simulators, the increase is much more important (up to $175 \mathrm{kbps})$. This result confirms that for OPNET and Qualnet, the implementation of the radio physical layer is not very realistic; the contentions are minimized which leads to a faster channel access when several sources compete. 


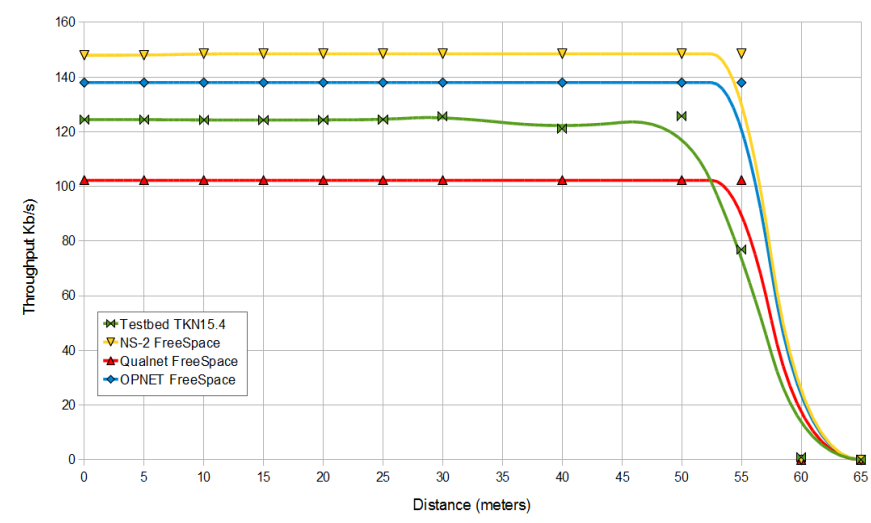

Figure 8. Throughput vs Distance Outdoor (Testbed and Simulators)

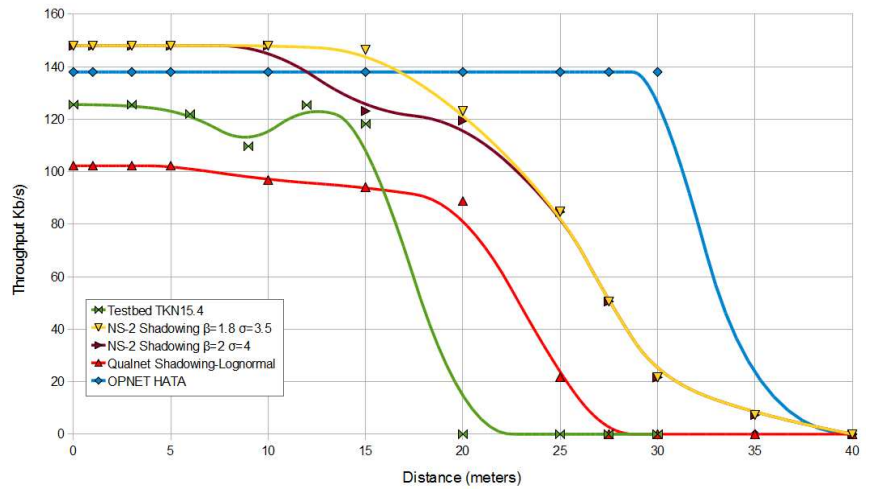

Figure 9. Throughput vs Distance Indoor (Testbed and Simulators)

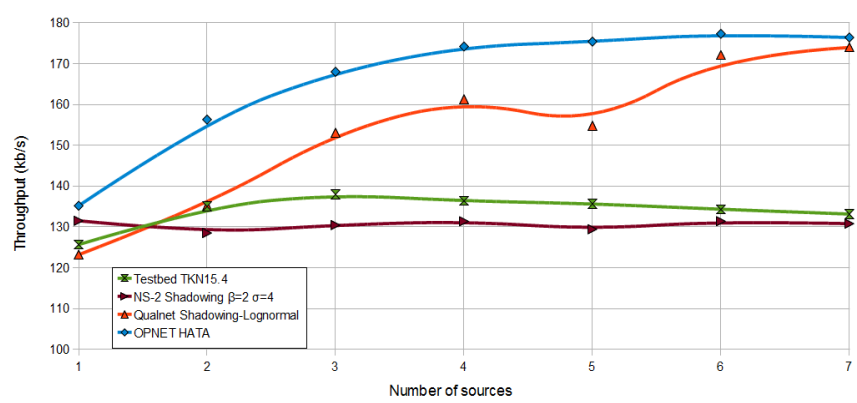

Figure 10. Throughput vs Number of sources (Testbed and Simulators)

\section{CONCLUSION}

In this work, we present a comparative study between MAC protocols which observe the IEEE 802.15.4 standard (such as TKN-15.4) and those which do not (such as BMAC). The impact of the different MAC protocols implementation in real testbed is presented and analyzed. In addition, a comparison between the results obtained from a real 802.15.4 testbed and three usual network simulators (NS-2, Qualnet and OPNET) is proposed. The main goals of this study are to test the performance of the real 802.15.4 MAC layers and to evaluate the relevance of the simulators, particularly in indoor and outdoor environments.

The 802.15.4 MAC layers implemented in the recent devices based on the CC2420 transceiver can be incomplete compared to the standard. The performances show that up to a certain range threshold, the throughput in both indoor and outdoor contexts is relatively constant, which confirms the robustness of the 802.15.4 channel. The lower layers of the simulators are approximate, do not include realistic propagation models and give a throughput that is not realistic. Finally, despite a difficult tuning of the propagation model parameters, NS-2 gives the results closest to reality in the indoor scenario, while OPNET gives the best results in the outdoor scenario.

In our future works, we plan to extend our study to the IEEE 802.15.4 beacon-enabled mode. In addition, improving the lower layers of the network simulators in order to generate results which are closer to the reality is another challenge.

\section{REFERENCES}

[1] Zigbee, "Zigbee Specification," ZigBee Standards Organization, Standard Zigbee 053474r17, January 2008

[2] IEEE 802.15, "Part 15.4: Wireless medium access control (MAC) and physical layer (PHY) specifications for low-rate wireless personal area networks (WPANs)," ANSI/IEEE, Standard 802.15.4 R2006, 2006.

[3] M. Petrova, J. Riihijarvi, P. Mahonen, S. Labella, "Performance study of IEEE 802.15.4 using measurements and simulations, IEEE Wireless Communications and Networking Conference," 2006. WCNC 2006.

[4] J-S Lee, "An experiment on performance study of IEEE 802.15.4 wireless networks," 10th IEEE Conference on .Emerging Technologies and Factory Automation, 2005. ETFA 2005.

[5] G. Lu, B. Krishnamachari, C.S. Raghavendra, "Performance Evaluation of the IEEE 802.15.4 MAC for Low-Rate Low-Power wireless Networks," IEEE International Performance Computing, and Communications Conference (IPCCC'2004)

[6] J. Zheng, M.J. Lee, "A comprehensive performance study of IEEE 802.15. 4, Sensor Network Operations,” IEEE Press, Wiley Interscience, Chapter 4, pp. 218-237, 2006. www-ee.ccny.cuny.edu

[7] A. Koubaa, M. Alves, E. Tovar, "A Comprehensive Simulation Study of Slotted CSMA/CA for IEEE 802.15.4 Wireless Sensor Networks," In Proc. 2006 IEEE International Workshop on Factory Communication Systems (WFCS), July 2006.

[8] I. Ramachandran, A.K. Das, S. Roy, "Analysis of the contention access period of IEEE 802.15.4 MAC," ACM Transactions on Sensor Networks (TOSN) Volume 3, Issue 1 (March 2007)

[9] J. Misic, S. Shaf, and V.B. Misic, "Performance of a Beacon Enabled IEEE 802.15.4 Cluster with Downlink and Uplink Traffic," IEEE Trans. Parallel and Distributed Systems, vol. 17, no. 4, pp. 361-376, Apr. 2006.

[10] C. K. Singh A. Kumar and P. M. Ameer, "Performance evaluation of an IEEE 802.15.4 sensor network with a star topology," Wireless Network Journal Published online: 21 August 2007

[11] Datasheet for Chipcon/Texas Instruments CC2420 $2.4 \mathrm{GHz}$ IEEE 802.15.4/ZigBee-Ready RF Tranceiver, http://focus.ti.com/lit/ds/symlink/cc2420.pdf [referenced September 2008].

[12] Samsung/CUNY, “Ns2 simulator for 802.15.4," http://wwwee.ccny.cuny.edu/zheng/pub [referenced September 13, 2005].

[13] MEMSIC Company http://www.memsic.com/products/wireless-sensornetworks.html

[14] The Network Simulator NS-2, http://www.isi.edu/nsnam/ns/

[15] OPNET http://www.opnet.com/solutions/network_rd/modeler.html

Modeler,

[16] QualNet, http://www.scalable-networks.com

[17] TinyOS 15.4 working group http://tinyos.stanford.edu:8000/15.4 WG

[18] J.-H. Hauer, "TKN15.4: An IEEE 802.15.4 MAC Implementation for TinyOS 2", TKN Technical Report Series TKN-08-003, Telecommunication Networks Group, Technical University Berlin, March 2009.

[19] J. Polastre, J. Hill, D. Culler, "Versatile low power media access for wireless sensor networks", In proceedings of the ACM 2nd international conference on Embedded networked sensor systems, 2004. 